\title{
Label-free identification of macrophage phenotype by fluorescence lifetime imaging microscopy
}

\author{
Alba Alfonso-García \\ Tim D. Smith \\ Rupsa Datta \\ Thuy U. Luu \\ Enrico Gratton \\ Eric O. Potma \\ Wendy F. Liu
}




\title{
Label-free identification of macrophage phenotype by fluorescence lifetime imaging microscopy
}

\author{
Alba Alfonso-García, ${ }^{a}$ Tim D. Smith, ${ }^{a}$ Rupsa Datta, ${ }^{a}$ Thuy U. Luu, ${ }^{b}$ Enrico Gratton, ${ }^{a}$ Eric O. Potma, ${ }^{c, *}$ and \\ Wendy F. Liu ${ }^{\mathrm{a}, *}$ \\ aUniversity of California Irvine, Department of Biomedical Engineering, 2412 Engineering Hall, Irvine, California 92697, United States \\ bUniversity of California Irvine, Department of Pharmacological Sciences, 2412 Engineering Hall, Irvine, California 92697, United States \\ 'University of California Irvine, Department of Chemistry, 2412 Engineering Hall, Irvine, California 92697, United States
}

\begin{abstract}
Macrophages adopt a variety of phenotypes that are a reflection of the many functions they perform as part of the immune system. In particular, metabolism is a phenotypic trait that differs between classically activated, proinflammatory macrophages, and alternatively activated, prohealing macrophages. Inflammatory macrophages have a metabolism based on glycolysis while alternatively activated macrophages generally rely on oxidative phosphorylation to generate chemical energy. We employ this shift in metabolism as an endogenous marker to identify the phenotype of individual macrophages via live-cell fluorescence lifetime imaging microscopy (FLIM). We demonstrate that polarized macrophages can be readily discriminated with the aid of a phasor approach to FLIM, which provides a fast and model-free method for analyzing fluorescence lifetime images. (c) 2016 Society of Photo-Optical Instrumentation Engineers (SPIE) [DOI: 10.1117/1.JBO.21.4.046005]
\end{abstract}

Keywords: macrophage; fluorescence lifetime imaging; phasor; nicotinamide adenine dinucleotides; glycolysis; oxidative phosphorylation; metabolism.

Paper 150788TNRR received Nov. 23, 2015; accepted for publication Mar. 15, 2016; published online Apr. $18,2016$.

\section{Introduction}

Macrophages exhibit a spectrum of functional phenotypes that are related to their role in the defense hierarchy of the body's immune system. They play a major role in advancing and resolving inflammation, ${ }^{1}$ which occurs in response to infections and during wound healing. At one end of this spectrum, in the first line of defense, macrophages exposed to inflammatory signals polarize toward a classically activated, inflammatory phenotype and secrete proinflammatory cytokines and reactive species. At the other end of the spectrum, macrophages present within a wound healing environment polarize toward an alternatively activated, prohealing phenotype and facilitate processes involved in tissue repair. ${ }^{2-4}$ In addition, macrophages play an important role in the numerous pathologies including cancer ${ }^{5,6}$ and cardiovascular disorders. ${ }^{5,7-9}$ Tumor-associated macrophages are thought to have an alternatively activated phenotype that provides immune privilege, facilitates tumor growth, and allows disease progression. ${ }^{2}$ In the cardiovascular system, macrophages ingest lipids that accumulate within the vessel wall, which leads to formation of foam cells and development of atherosclerotic lesions. ${ }^{7,10,11}$ Therefore, methods to distinguish different macrophage phenotypes are important for basic biological studies, and may also be beneficial for diagnosis of diseases in which macrophages are involved.

Traditionally, the identification of macrophage subpopulations has relied heavily on quantifying the expression of proinflammatory and prohealing markers through immunolabeling or analysis of gene expression. Common markers include inducible nitric oxide synthase (iNOS) and arginase- $1(\operatorname{Arg} 1)^{5,6}$ or surface markers such as $\mathrm{CD} 6^{5,7-9}$ and CD206, ${ }^{12,13}$ which report on the

*Address all correspondence to: Eric O. Potma, Email: epotma@uci.edu; Wendy F. Liu, Email: wendy.liu@uci.edu proinflammatory and the prohealing state of the cell, respectively. Antibody- or nucleic acid-mediated interrogation of the cells' state are widespread techniques, but typically require elaborate biochemical protocols that contain many steps. ${ }^{7,14-17}$ These techniques are generally not capable of assessing live cells in their native environments, since cells are detached from the surface, lysed, and/or fixed. In order to assess the dynamics of phenotypic changes of cells during the course of complex cellular studies/treatments, it would be desirable to employ a method that is based on endogenous markers and therefore compatible with live cell and tissue imaging. Given the role of macrophages in the pathogenesis of many diseases, methods to image their phenotype within live animals will also be beneficial.

As a reflection of their distinct functions, classically and alternatively activated macrophages have been shown to differ in their metabolic state. While classically activated macrophages produce energy via aerobic glycolysis, alternatively activated macrophages obtain energy from fatty acid oxidation and undergo oxidative phosphorylation. ${ }^{4,18}$ These pathways produce reduced nicotinamide adenine dinucleotides (NADH) with different binding properties: freely diffusing NADH is a product of glycolysis, ${ }^{19}$ whereas NADH binds to mitochondrial shuttles (i.e., malate-aspartate) to undergo oxidation. ${ }^{20}$ The binding state of NADH in the cells translates into different fluorescence lifetimes of this autofluorescent coenzyme [free $(\sim 0.4 \mathrm{~ns})$ or bound ( 2 to $4 \mathrm{~ns}$ )], principally due to the quenching state of adenine. $^{21}$ Therefore, fluorescence lifetime analysis can gauge the metabolic state of the cell, and may be used to assess macrophage phenotype in live cells and in a label-free manner. 
Here, we present a label-free method capable of recognizing macrophage subpopulations of living cells. This method may be used in conjunction with a recently reported label-free technique that distinguishes inflammatory and healing macrophages based on their differences in angular light scattering. ${ }^{22}$ Our method is based on two-photon fluorescence lifetime imaging microscopy (FLIM) of NADH, and can readily be extended to in vivo studies, which is not feasible for light-scattering measurements. In addition, the phasor approach to FLIM simplifies the data analysis process to the visual inspection of clusters, especially if measured lifetimes arise from complex multiexponential decays. $^{23}$ In such cases, the commonly used direct fitting approach may prove challenging. However, in the phasor plot representation, the lifetimes emerge as clustered distributions that can be assigned directly to different macrophage phenotypes, a technique previously reported for the metabolic state of germ cells ${ }^{24}$ or neural stem cells. ${ }^{25} \mathrm{NADH}$ quantification through the redox ratio has also been employed to determine stem cell differentiation. ${ }^{26}$ In the cells' cytoplasm, a combination of freely diffusing and bound NADH is found. When excited at the correct wavelength, and in the absence of other exogenous or exotic fluorescent components, $\mathrm{NADH}$ is the main autofluorescent species that contributes to the optical emission, yielding a linear combination of the single exponential decays of free and bound NADH in the phasor plot. ${ }^{21,27} \mathrm{We}$ demonstrate that this approach allows the identification of macrophage phenotype in cell populations and that this method retains its robustness and ease of use under a variety of conditions.

\section{Methods}

\subsection{Cell Culture}

Mouse bone marrow-derived macrophages (BMDM) were isolated from femurs of female, 6- to 12-week-old C57BL/6J mice and cultured as described ${ }^{28}$ in medium containing $10 \%$ fetal bovine serum (FBS), $100 \mathrm{U} / \mathrm{ml}$ penicillin, $100 \mu \mathrm{g} / \mathrm{ml}$ streptomycin, and $10 \%$ media conditioned by Ltk-cells expressing recombinant MCSF ectopically. On day 7, $3 \times 10^{5} \mathrm{BMDMs}$ were seeded on glass coverslips. The following day, cells were transferred to similar media containing either lipoproteindepleted bovine serum (LPDS; Alfa Aesar) or normal FBS and then stimulated with cytokines. Lipopolysaccharides (LPS; Sigma) and interferon- $\gamma$ (IFN- $\gamma ; \mathrm{R} \& \mathrm{D})$ were used at $10 \mathrm{ng} / \mathrm{ml}$, and IL-4 and IL-13 (Biolegend) were used at $20 \mathrm{ng} / \mathrm{ml}$. After $24 \mathrm{~h}$ of cytokine stimulation, media was replaced (with lipoprotein-depleted or normal media, per the experimental condition) and cells were exposed to 0 or $40 \mu \mathrm{g} / \mathrm{ml}$ oxidized human low-density lipoprotein (Alfa Aesar) for the remaining duration of the experiment.

\subsection{Immunofluorescence Staining}

Samples were fixed in $100 \%$ methanol and washed with $1 \%$ bovine serum albumin (BSA; MP Biomedical) in PBS. Cells were blocked with 5\% normal donkey serum and stained for arginase-1 with a goat polyclonal primary antibody (1:50; Santa Cruz) and Alexa 594-conjugated donkey anti-goat IgG (1:500; Jackson ImmunoResearch). After washing in 1\% BSA, cells were blocked a second time with $5 \%$ normal goat serum and stained for iNOS with a rabbit polyclonal primary antibody (1:50; Santa Cruz) and Alexa 488-conjugated goat anti-rabbit
IgG (1:200; Jackson ImmunoResearch). Nuclei were visualized with Hoechst 33342.

\subsection{Fluorescence Lifetime Imaging Microscopy, Data Acquisition, and Processing}

Fluorescence lifetime images were acquired with a Zeiss LSM 710 microscope (Carl Zeiss, Jena, Germany). A Titanium: Sapphire MaiTai laser (Spectra-Physics, Mountain View, California) with $100 \mathrm{fs}$ pulses and $80 \mathrm{MHz}$ repetition rate was used as a two-photon excitation source at $740 \mathrm{~nm}$. The laser light was focused through a $40 \times, 1.2$ N.A., water immersion objective (Carl Zeiss, Oberkochen, Germany). The autofluorescence was detected with a photomultiplier tube (H7422P-40, Hamamatsu, Japan) after passing through a bandpass $460 / 80 \mathrm{~nm}$ filter. A dichroic filter at $690 \mathrm{~nm}$ served to separate the excitation from the emission signals. Fluorescence lifetime data were acquired using an A320 FastFLIM FLIMbox (ISS, Champaign, Illinois). For each image, 60 frames were collected to obtain at least 100 photons/pixel at $3 \mathrm{~mW}$ of power in the sample plane. The scan speed was $25.21 \mu \mathrm{s} /$ pixel, and the image size $256 \times 256$ pixels, which span across $154 \mu \mathrm{m}$. The lifetime of rhodamine 110 was measured to calibrate the FLIM system, as it is established at 4 ns.

The SimFCS software, developed at the Laboratory of Fluorescence Dynamics (UC Irvine), was used to collect and process these FLIM data. As previously described, ${ }^{23,29}$ the fluorescence intensity decay associated to each pixel of the FLIM image is mapped onto a two coordinates $(g, s)$ system, the socalled phasor plot. The phasor transformation is as follows:

$$
\begin{aligned}
& g_{i, j}(\omega)=\int_{0}^{\infty} I_{i, j}(t) \cos (\omega t) \mathrm{d} t / \int_{0}^{\infty} I_{i, j}(t) \mathrm{d} t \\
& s_{i, j}(\omega)=\int_{0}^{\infty} I_{i, j}(t) \sin (\omega t) \mathrm{d} t / \int_{0}^{\infty} I_{i, j}(t) \mathrm{d} t
\end{aligned}
$$

where $\omega$ is the laser repetition rate, $I(t)$ is the fluorescence intensity decay, and the indices $i$ and $j$ identify each pixel of the image. After the transformation, the data are represented as clusters of lifetime distributions in the phasor plot, also known as phasors. These lifetime distributions (or phasors) are either attributed to single exponential decays, which are located along the periphery of a semicircle, or to multiple exponential decays, placed within the semicircle. In the phasor space, a lifetime distribution attributed to multiple exponential decays is a linear combination of the lifetime distributions associated with the single exponential decays that constitute the combination. ${ }^{23}$ This property simplifies the analysis and provides a fitting-free approach for identifying areas in the image with similar or distinct lifetimes.

\subsection{Statistical Analysis}

Statistical significance between the phasors of the examined macrophage groups was determined by the Student's $t$-test, and considered positive for $p<0.05$. The analysis was performed on 3 to 7 images per each group as indicated in each experiment. Cells from at least two separate mice were obtained, and a total of 12 to 32 cells were analyzed for each condition. 

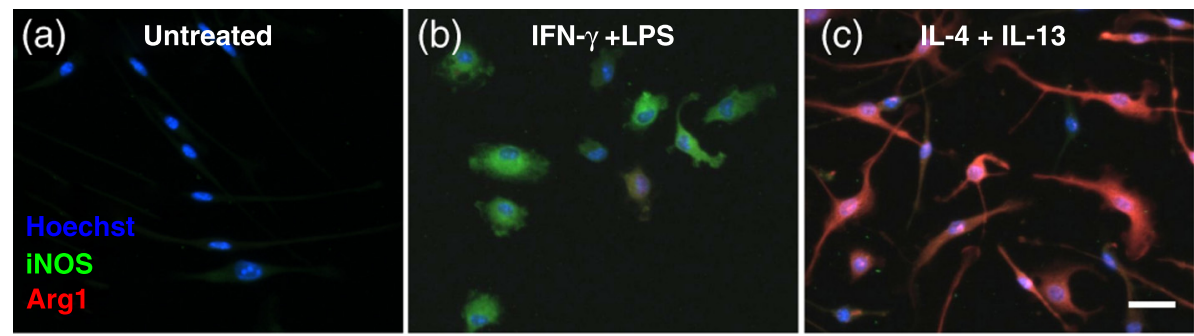

Fig. 1 Immunofluorescence staining of macrophage markers. Representative immunofluorescence images of macrophages polarized with (b) IFN- $\gamma$ and LPS or (c) IL-4 and IL-13, or (a) untreated, and stained for the proinflammatory marker iNOS (in green), the prohealing marker arginase-1 (Arg1, in red), and the cell nuclei (Hoechst 33342, in blue). Macrophages polarized with IFN- $\gamma$ and LPS express higher levels of iNOS, while macrophages polarized with IL-4 and IL-13 express predominantly Arg1. Scale bar $=25 \mu \mathrm{m}$.

\section{Results}

\subsection{Fluorescence Lifetime Imaging Microscopy of Free and Bound NADH in Unpolarized Macrophages}

It is well established that macrophages stimulated with IFN- $\gamma$ and LPS or with interleukins 4 and 13 (IL-4 and IL-13) render proinflammatory or prohealing polarization states, respectively. ${ }^{7,30,31}$ We confirm this by immunofluorescence staining for the proinflammatory marker inducible nitric oxide synthase (iNOS, in green) and the prohealing marker arginase-1 (Arg1, in red) (Fig. 1). IFN- $\gamma /$ LPS-polarized macrophages express higher levels of iNOS, while IL-4/IL-13stimulated macrophages express predominantly Arg1, as expected. Nuclei were counterstained with Hoechst 33342, indicated in blue.

Before interrogating the polarized macrophages with FLIM, we first study NADH lifetime maps of unpolarized macrophages that we will use as the reference. Figure 2(a) shows an autofluorescence image of untreated macrophages, and Fig. 2(b) shows

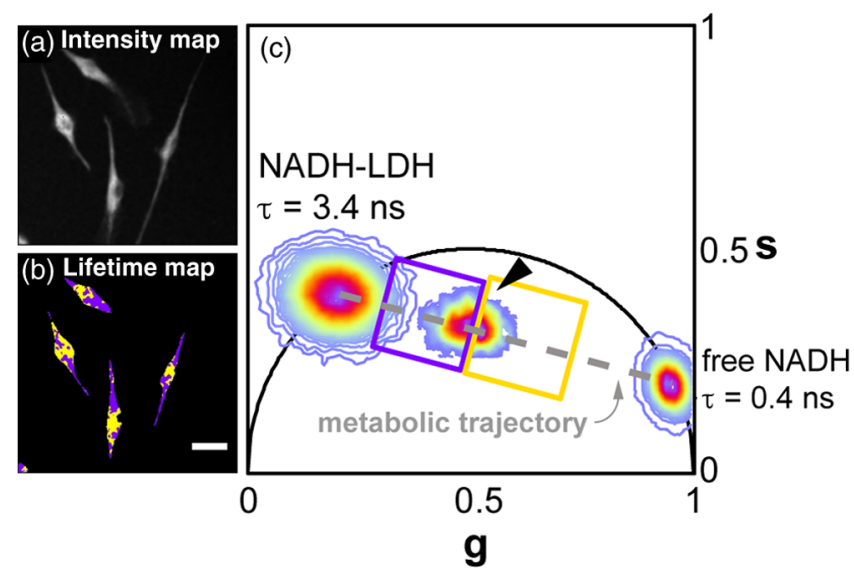

Fig. 2 NADH lifetime of unpolarized macrophages. (a) Autofluorescence intensity images of untreated macrophages. (b) Corresponding lifetime image with color code according to squares selected in (c): purple areas have a more bound NADH character, while yellow areas have a more free $\mathrm{NADH}$ character (scale bar $=25 \mu \mathrm{m}$ ). (c) Phasor plots of NADH bound to LDH, free NADH, and the cells in image $a / b$ (arrowhead). The line that joins the free with the bound NADH phasors, centered at 0.4 and 3.4 ns lifetimes, respectively, defines the metabolic trajectory. The cells' phasor lies along this line, as a linear combination of the two lifetimes. a map based on lifetime contrast. The color coding is derived from the phasor plot in Fig. 2(c). In this plot, the lifetime distributions of pure free NADH and NADH bound to lactate dehydrogenase $(\mathrm{LDH})$ are indicated. The two distributions are found at opposite sides of the unit semicircle, also known as the universal circle, ${ }^{23}$ which starts at point $(1,0)$ for zero lifetime species, and continues on to point $(0,0)$ for infinite lifetime species. Freely diffusing NADH has a lifetime of $\sim 0.4$ ns and NADH bound to LDH has a longer lifetime of $\sim 3.4 \mathrm{~ns}$, the latter varies slightly with binding protein. Both lifetimes are characterized by a single exponential decay and therefore lie on the semicircle. ${ }^{32}$

The two NADH states define the extremes of the so-called metabolic trajectory in the phasor plot. ${ }^{33,34}$ In the cellular cytoplasm, we find both species at different ratios, which yield a lifetime distribution that falls along the metabolic trajectory [Fig. 2(c), arrowhead]. This lifetime distribution (or phasor) can be characterized as the linear combination of the free NADH and bound NADH phasors. The phasor approach to FLIM permits a rapid identification of changes in the lifetime distributions. $^{23}$ The metabolic state of the cell can be determined by the position of the phasor along the metabolic trajectory. We divide the lifetime distribution of untreated macrophages through the center of mass in two parts along the metabolic trajectory, so that the fraction of pixels in each section is $0.5 \pm 10 \%$. The left part contains the pixels that are closer to the bound NADH extreme of the metabolic trajectory (purple square), while the right part has those closer to the free NADH end (yellow square). In this two-color representation, each pixel in the lifetime image in Fig. 2(b) is presented as either purple or yellow. The color coding can be interpreted as a relative measure of bound versus free NADH: purple represents more bound character, a lower free to bound NADH ratio, and yellow represents less bound character, a higher free to bound NADH ratio. Using these analysis regions as a reference, we can examine how the phasors of the polarized macrophages move along the metabolic trajectory, and thus quantify the changes in the ratio of free to bound NADH for each group.

\subsection{Fluorescence Lifetime Imaging Microscopy Identifies Different Lifetime Distributions in Polarized Macrophages}

Using this approach, we next examine NADH lifetime distributions of polarized macrophages. Figure 3(a) shows an image with lifetime contrast obtained from unpolarized cells, and the accompanying color scheme is defined in the phasor plot of Fig. 3(b). As before, the cells display different color regions, 

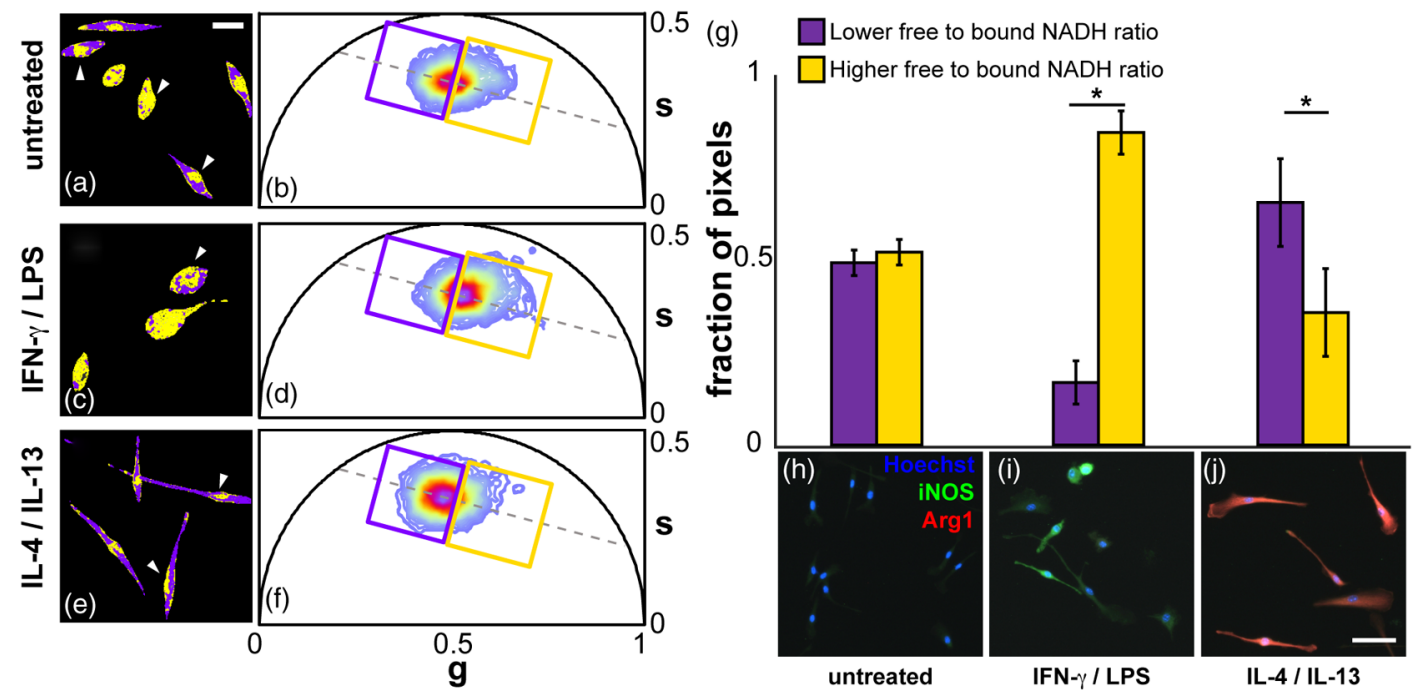

Fig. 3 Polarized macrophages have different lifetime distributions. (a) Untreated macrophages lifetime map (scale bar $=25 \mu \mathrm{m}$ ), and (b) corresponding phasor plot. The purple and yellow squares select the areas in the cells with more bound NADH character, and more free NADH character, respectively, and are kept constant throughout the entire analysis. (c) Inflammatory macrophages, polarized with IFN- $\gamma$ and LPS, and (d) corresponding phasor plot, shifted toward a markedly free NADH character. (e) Healing macrophages, polarized with IL-4 and IL-13, and (f) corresponding phasor plot, shifted toward the bound NADH side of the plot. (g) Average fraction of pixels in each square, normalized to the total number of pixels in both squares, for the three macrophage cultures, the error bars correspond to the standard deviation. $* p<0.05$, Student $t$-test. (h) Immunofluorescence images of the untreated macrophage culture, (i) the IFN- $\gamma$ - and LPS-polarized culture, and (j) the IL-4/IL-13-polarized culture stained for iNOS (green), Arg1 (red), and nuclei using Hoechst 33342 (blue). Scale bar $=50 \mu \mathrm{m}$.

corresponding to relative changes in the fluorescence lifetime, although the shape of the overall lifetime distribution may vary slightly from analysis of different cell populations. Using the same definition of the lifetime contributions as in Fig. 3(b), Fig. 3(c) shows a lifetime image of cells treated with IFN- $\gamma$ and LPS, corresponding to proinflammatory macrophages. Compared to untreated cells, the IFN- $\gamma /$ LPS cells have a phasor slightly shifted toward the free NADH end of the trajectory [Fig. 3(d)], which translates into larger yellow areas in the cells [Fig. 3(c)]. This is expected, as IFN- $\gamma /$ LPS macrophages turn on glycolytic pathways for energy generation. ${ }^{35}$ In contrast, cells treated with IL-4 and IL-13 have a higher contribution from bound NADH, as indicated in purple, as shown in Fig. 3(e). The phasor plot in Fig. 3(f) is now shifted toward the direction of pure bound NADH, with effectively longer lifetimes. This observation is consistent with the notion that alternatively activated macrophages favor oxidative phosphorylation, ${ }^{36}$ which requires protein-bound NADH. ${ }^{19}$ IL-4/IL-13-treated macrophages show a markedly elongated profile compared to IFN- $\gamma$ / LPS and unpolarized macrophages, this is expected as macrophage elongation has been previously related to an alternatively activated phenotype. $^{16}$

In an effort to quantify the metabolic state of the cells, we compute the fraction of pixels in each image that fall into the predefined lifetime categories (indicated here by the purple and yellow squares in the phasor plot). Figure 3(g) shows the results of averaging the fraction of pixels (fop) over three images for the unpolarized cells, with a total of 14 cells from two different mice, and over seven images for the polarized groups, that contained a total of 21 and 32 cells for each inflammatory and healing macrophages, respectively, obtained from three separate animals. Untreated macrophages show roughly equal amounts of short and longer lifetimes, as defined by the chosen purple (fop lower free to bound $\mathrm{NADH}$ ratio $=0.49 \pm 0.03$ ) and yellow (fop higher free to bound $\mathrm{NADH}$ ratio $=0.51 \pm 0.03$ ) subgroups [Figs. 3(a) and 3(b)]. However, macrophages polarized with IFN $-\gamma$ and LPS have a significant increase in the fraction of yellow pixels (fop higher free to bound NADH ratio $=0.83 \pm 0.06$ versus fop $_{\text {lower free to bound NADH ratio }}=0.17 \pm 0.06$ ), those that report an increase in glycolytic metabolism. These cells are depicted mainly as yellow in Fig. 3(c). Macrophages treated with IL-4 and IL-13 have the opposite behavior, the fraction of pixels within the purple square (fop lower free to bound $\mathrm{NADH}$ ratio $=0.65 \pm 0.12$ ) is significantly larger than those in the yellow square (fop figher free to bound NADH ratio $=0.35 \pm 0.12$ ). As a result, the cells are colored predominantly in purple in Fig. 3(e). After FLIM imaging, the cells were fixed and stained to examine their expression levels of proinflammatory and prohealing markers [Figs. 3(h)-3(j)]. The stained cells confirm the correlation between NADH metabolism and macrophage phenotype, as IFN- $\gamma /$ LPS-treated cells have higher expression of iNOS [Fig. 3(i)] and Arg1 expression predominates in IL-4/IL-13treated cells [Fig. 3(j)].

In addition to evaluating the aggregate response of a population of cells with the overall lifetime distribution, the phasor approach to FLIM also permits the visualization of intracellular features with different lifetime properties. More specifically, the nuclear regions in the cells tend to have a higher free NADH concentration (as shown in yellow) in the lifetime images [white arrowheads in Figs. 3(a), 3(c), 3(e)], whereas the cellular cytoplasm displays a higher concentration of bound NADH (as shown in purple). A shift toward free NADH in the nuclei has been previously reported using this same method to identify early stages of cellular differentiation. ${ }^{37}$

In addition to a rapid visualization method, the phasor approach can be used to classify the cells according to their 


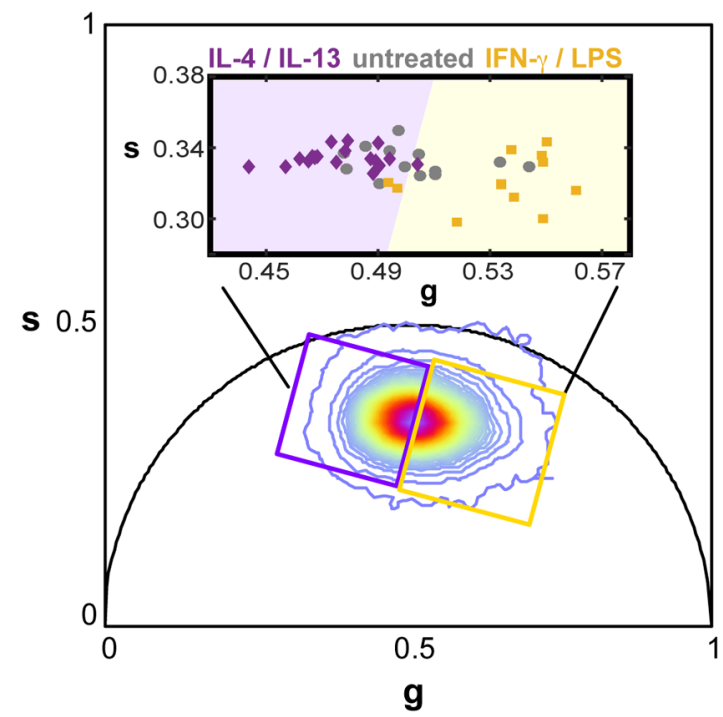

Fig. 4 Cell phasor plots. The phasor plot agglutinates the lifetime distributions of the cells in the untreated and the polarized macrophages. The purple and yellow squares are located in the same position for the entire analysis. The insert zooms into the core of the phasor to show the average phasor point of each cell analyzed across separate macrophage cultures. The shaded areas align with the squares position.

average individual phasor value. In that regard, Fig. 4 shows the aggregate phasor distribution of untreated and polarized macrophages, and locates the position of single cells analyzed within the distribution. The purple and yellow squares are kept constant and divide the zoomed-in area in shaded purple and yellow regions for reference. We find two clear groups, the IL-4/IL-13-polarized macrophages (purple diamonds) fall within the purple square, with higher contribution of proteinbound NADH, and the IFN- $\gamma /$ LPS-polarized macrophages (yellow squares) fall into the yellow area with lower lifetimes, assigned to higher contribution of free NADH. Unpolarized macrophages stretch across the areas, with a skewed preference toward the left side of the plot, indicative of an oxidative metabolism basal state. ${ }^{14,35}$

\subsection{NADH Lifetime Detected by FLIM is a Robust Indicator of Macrophage Glycolytic State}

Given that macrophages are involved in the progression of cardiovascular disease, a condition in which the cellular microenvironment contains significantly more lipid, we were interested to know whether or not the presence of lipid influenced the polarization of macrophages as determined by FLIM. We interrogated polarized macrophages with low (lipoprotein deficient serum, LPDS, based medium) or excess (oxidized low-density lipoprotein, oxLDL, containing medium) lipid. None of the cells in these conditions appeared to accumulate significant lipid droplets (data not shown). We find the same results: inflammatory macrophages (cells polarized with IFN- $\gamma$ and LPS) have a significantly larger fraction of glycolytic pixels in both cases; whereas healing macrophages (cells polarized with IL-4 + IL-13) significantly bind more NADH to protein to undergo oxidative phosphorylation than inflammatory cells do [Fig. 5(i)], independent of the lipid environment. The fraction of pixels quantified in [Fig. 5(i)] are the average 5 to 7 images that contained a total of 12 to 23 macrophages from two separate mice. IFN- $\gamma /$ LPS-stimulated macrophages treated with LPDS ( $N=6$ images, 12 cells) have in average a fop lower free to bound $\mathrm{NADH}$ ratio $=0.27 \pm 0.14$ and fop phigher free to bound NADH ratio $_{1}=0.73 \pm 0.14$, while IL-4/IL13stimulated macrophages treated with LPDS $(N=5$ images, 23 cells) have in average a fop lower free to bound $\mathrm{NADH}$ ratio $=$ $0.57 \pm 0.05$ and fop higher free to bound $\mathrm{NADH}$ ratio $=0.43 \pm 0.05$. Macrophages stimulated with oxLDL followed the same trend, the average fraction of pixels and standard deviation for IFN- $\gamma /$ LPS-stimulated macrophages $(N=7$ images, 15 cells) are fop lower free to bound NADH ratio $=0.24 \pm$ 0.10 and fop $\mathrm{p}_{\text {higher free to bound NADH ratio }}=0.76 \pm 0.10$, and for IL-4/IL13-stimulated macrophages $(N=5$ images,
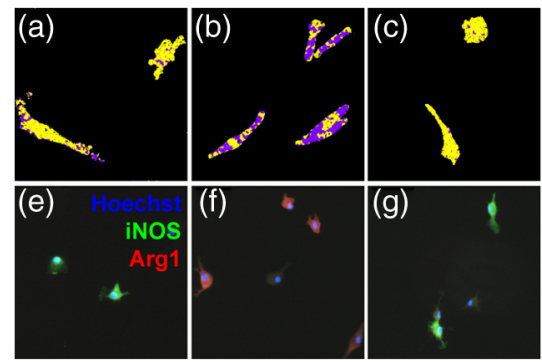

IFN- $\gamma$ / LPS

IL-4 /IL-13

IFN- $\gamma$ / LPS

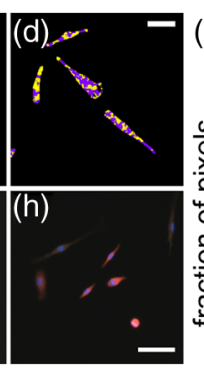

IL-4 / IL-13

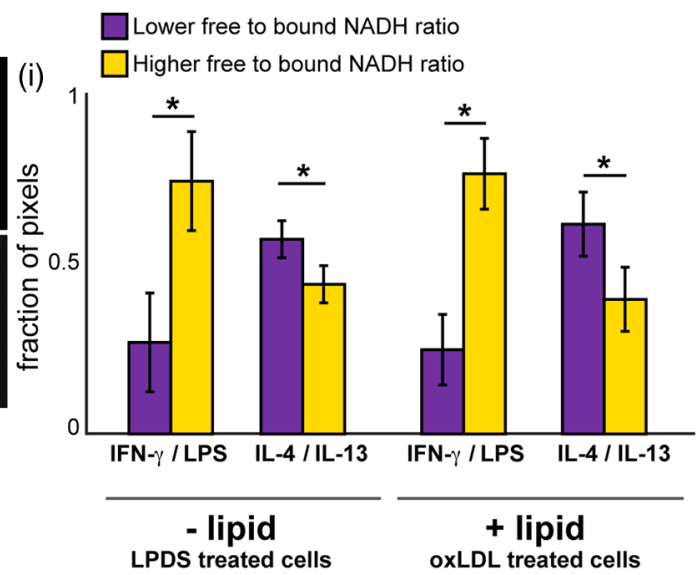

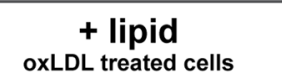

- lipid
LPDS treated cells

oxLDL treated cells

Fig. 5 Macrophages under altered lipid uptake conditions. Macrophages polarized with IFN- $\gamma$ and LPS and macrophages polarized with IL-4 and IL-13 treated with LPDS and with oxLDL maintain their glycolytic phenotype. (a-d) Lifetime maps (scale bar $=25 \mu \mathrm{m})$ and $(e-h)$ immunofluorescence images of the corresponding macrophage groups stained for iNOS (green), Arg1 (red), and nuclei with Hoechst 33342 (blue). Scale bar $=50 \mu \mathrm{m}$. (i) Average fraction of pixels in the yellow and purple squares, normalized to the total fraction of pixels, for each group of macrophage culture, the error bars correspond to the standard deviation. $* p<0.05$, Student $t$-test. 
18 cells) are fop lower free to bound NADH ratio $=0.61 \pm 0.09$ and fop $_{\text {higher free to bound NADH ratio }}=0.39 \pm 0.09$. Once again this is visually translated at the cellular level by yellow in the IFN- $\gamma /$ LPS cases [Figs. 5(a) and 5(c)], and purple in the IL-4/IL13 cases [Figs. 5(b) and 5(d)]. We further confirmed by immunofluorescence staining that cells in these conditions maintained their phenotypic markers, as shown in Figs. 5(e)5(h). Thus, this method appears robust to alterations in the presence of different lipid microenvironments.

\section{Conclusion}

NADH fluorescence lifetime imaging microscopy provides a label-free, nondestructive means to identify the phenotype of live macrophages. FLIM indicates the metabolic state of polarized macrophages; whether the cells shift to a glycolytic state or rather switch to an oxidative metabolism, corresponding to a classically activated versus an alternatively activated phenotype, respectively.

Immunoassays or genetic-based assays to detect phenotypic markers are the standard techniques used to identify macrophage polarization states, but typically require cells to be fixed or lysed and to undergo an elaborate staining protocol. The FLIM-phasor approach presented here allows characterization based on endogenous labels, which requires no sample preparation, and, most importantly, permits imaging of live cells directly in tissues. While this is not definitive to pinpoint the exact phenotype of a particular macrophage, as inflammatory and healing are extreme states, and to completely identify the exact phenotype of macrophages (especially in situ) multiple tests are recommended, ${ }^{14}$ FLIM and the phasor approach provide an effective threshold to differentiate between glycolytic and oxidative metabolisms. In addition, this method provides the ability to examine the metabolic state of the cells in realtime, allowing for a fast identification of macrophage state. This is particularly advantageous in dynamic studies that require controlled tracking of the phenotype as a given treatment evolves. Furthermore, since macrophages play a critical role in many diseases including cancer and cardiovascular disease, ${ }^{11,38}$ noninvasive imaging tools to evaluate macrophage behavior during the progression of disease may provide critical insight toward understanding their role in pathogenesis.

\section{Acknowledgments}

This work was supported by the following grants from the National Institutes of Health (NIH): DP2DE023319 (WFL), P41-RR01192 (Laser Microbeam and Medical Program, LAMMP) (EOP), and P41-GM103540 and P50-GM076516 (EG). TDS is supported by a NSF GRFP fellowship (DGE1321846).

\section{References}

1. T. Wynn and L. Barron, "Macrophages: master regulators of inflammation and fibrosis," Semin. Liver Dis. 30(03), 245-257 (2010).

2. J. W. Pollard, "Tumour-educated macrophages promote tumour progression and metastasis," Nat. Rev. Cancer 4, 71-78 (2004).

3. D. M. Mosser and J. P. Edwards, "Exploring the full spectrum of macrophage activation," Nat. Publ. Group 8(12), 958-969 (2008).

4. L. A. J. O. Silvia Galván-Peña, "Metabolic reprograming in macrophage polarization," Front. Immunol. 5, 420 (2014).

5. J. L. Stöger et al., "Distribution of macrophage polarization markers in human atherosclerosis," Atherosclerosis 225, 461-468 (2012).

6. A. Mantovani et al., "Cancer-related inflammation," Nature 454(7203), 436-444 (2008).
7. K. J. Moore, F. J. Sheedy, and E. A. Fisher, "Macrophages in atherosclerosis: a dynamic balance," Nat. Rev. Immunol. 13, 709-721 (2013).

8. D. M. Mosser, "The many faces of macrophage activation," J. Leukocyte Biol. 73, 209-212 (2003).

9. B. Legein et al., "Inflammation and immune system interactions in atherosclerosis," Cell. Mol. Life Sci. 70, 3847-3869 (2013).

10. F. H. Epstein and R. Ross, "Atherosclerosis-an inflammatory disease," N. Engl. J. Med. 340, 115-126 (1999).

11. K. J. Moore and I. Tabas, "Macrophages in the pathogenesis of atherosclerosis," Cell 145(3), 341-355 (2011).

12. M. A. Bouhlel et al., "PPAR $\gamma$ activation primes human monocytes into alternative M2 macrophages with anti-inflammatory properties," Cell Metab. 6(2), 137-143 (2007).

13. G. Chinetti-Gbaguidi et al., "Human atherosclerotic plaque alternative macrophages display low cholesterol handling but high phagocytosis because of distinct activities of the PPAR and LXR pathways," Circulation Res. 108(8), 985-995 (2011).

14. R. Orihuela, C. A. McPherson, and G. J. Harry, "Microglial M1/M2 polarization and metabolic states," Br. J. Pharmacol. 173, 649-665 (2015).

15. U. Muller et al., "IL-13 induces disease-promoting type 2 cytokines, alternatively activated macrophages and allergic inflammation during pulmonary infection of mice with cryptococcus neoformans," J. Immunol. 179(8), 5367-5377 (2007).

16. F. Y. McWhorter et al., "Modulation of macrophage phenotype by cell shape," Proc. Natl. Acad. Sci. U. S. A. 110(43), 17253-17258 (2013).

17. S. Fujisaka et al., "Regulatory mechanisms for adipose tissue M1 and M2 macrophages in diet-induced obese mice," Diabetes 58(11), 2574$2582(2009)$

18. S. K. Biswas and A. Mantovani, "Orchestration of metabolism by macrophages," Cell Metab. 15(4), 432-437 (2012).

19. K. Eto, "Role of NADH shuttle system in glucose-induced activation of mitochondrial metabolism and insulin secretion," Science 283(5404), 981-985 (1999).

20. M. C. McKenna et al., "Neuronal and astrocytic shuttle mechanisms for cytosolic-mitochondrial transfer of reducing equivalents: current evidence and pharmacological tools," Biochem. Pharmacol. 71(4), 399-407 (2006).

21. J. R. Lakowicz et al., "Fluorescence lifetime imaging of free and protein-bound NADH," Proc. Natl. Acad. Sci. U. S. A. 89(4), 1271-1275 (1992).

22. D. L. Halaney et al., "Differences in forward angular light scattering distributions between M1 and M2 macrophages," J. Biomed. Opt. 20(11), 115002 (2015).

23. M. A. Digman et al., "The phasor approach to fluorescence lifetime imaging analysis," Biophys. J. 94(2), L14-L16 (2008).

24. C. Stringari et al., "Phasor approach to fluorescence lifetime microscopy distinguishes different metabolic states of germ cells in a live tissue," Proc. Natl. Acad. Sci. U. S. A. 108(33), 13582-13587 (2011).

25. C. Stringari et al., "Phasor fluorescence lifetime microscopy of free and protein-bound NADH reveals neural stem cell differentiation potential," PLOS ONE 7(11), e48014 (2012).

26. K. P. Quinn et al., "Quantitative metabolic imaging using endogenous fluorescence to detect stem cell differentiation," Sci. Rep. 3, 3432 (2013).

27. M. C. Skala et al., "In vivo multiphoton microscopy of NADH and FAD redox states, fluorescence lifetimes, and cellular morphology in precancerous epithelia," Proc. Natl. Acad. Sci. U. S. A. 104(49), 19494-19499 (2007).

28. P. Morgado et al., "Toxoplasma gondii induces B7-2 expression through activation of JNK signal transduction," Infect. Immun. 79(11), 44014412 (2011).

29. R. A. Colyer, C. Lee, and E. Gratton, "A novel fluorescence lifetime imaging system that optimizes photon efficiency," Microsc. Res. Tech. 71(3), 201-213 (2008).

30. J. Ma et al., "Regulation of macrophage activation," Cell. Mol. Life Sci. 60(11), 2334-2346 (2003).

31. L. L. Stoll, "Potential role of endotoxin as a proinflammatory mediator of atherosclerosis," Arteriosclerosis Thrombosis Vasc. Biol. 24(12), 2227-2236 (2004). 
32. G. I. Redford and R. M. Clegg, "Polar plot representation for frequencydomain analysis of fluorescence lifetimes," J. Fluoresc. 15(5), 805-815 (2005).

33. C. Stringari et al., "Metabolic trajectory of cellular differentiation in small intestine by Phasor fluorescence lifetime microscopy of NADH," Sci. Rep. 2, 568 (2012).

34. R. Datta et al., "Fluorescence lifetime imaging of endogenous biomarker of oxidative stress," Sci. Rep. 5, 9848 (2015).

35. J.-C. Rodríguez-Prados et al., "Substrate fate in activated macrophages: a comparison between innate, classic, and alternative activation," J. Immunol. 185, 605-614 (2010).

36. D. Vats et al., "Oxidative metabolism and PGC-1 $\beta$ attenuate macrophage-mediated inflammation," Cell Metab. 4, 13-24 (2006).

37. B. K. Wright et al., "Phasor-flim analysis of NADH distribution and localization in the nucleus of live progenitor myoblast cells," Microsc. Res. Tech. 75(12), 1717-1722 (2012)

38. A. Sica and A. Mantovani, "Macrophage plasticity and polarization: in vivo veritas," J. Clin. Invest. 122(3), 787-795 (2012).

Alba Alfonso-García is a PhD student in biomedical engineering at the University of California, Irvine. She is interested in applying nonlinear optical techniques to image biological processes, with a particular focus on lipid metabolism.

Tim D. Smith is a PhD student in biomedical engineering at the Unversity of California, Irvine. His research interests include the study of macrophage-material interactions.

Rupsa Datta is a PhD student in the Laboratory of Fluorescence Dynamics, University of California, Irvine. Her research entails application of fluorescence lifetime imaging microscopy to study metabolism and oxidative stress in biological systems including cells, tissues and microfluidic device-based micro-physiological tissue systems.

Thuy U. Luu is a PhD student in the Department of Pharmacological Sciences at the University of California, Irvine. Her research is focused on investigating the effect of surface topographies on macrophage cell shape and function, and elucidating the intracellular mechanism by which macrophages sense topography, and transduce physical cues to changes in phenotype.

Enrico Gratton in 1986 was awarded a grant from NIH to establish the first national facility dedicated to fluorescence spectroscopy: the Laboratory for Fluorescence Dynamics (LFD). Since then the LFD has achieved international recognition for the development of instrumentation for time-resolved fluorescence spectroscopy using frequency domain methods. In 2006, the LFD laboratory moved at UCl where the LFD has continued to be at the forefront of dynamics approaches using fluorescence spectroscopy and imaging.

Eric O. Potma is an associate professor in the Department of Chemistry at the University of California, Irvine. He holds an adjunct position in the Beckman Laser Institute and Medical Clinic at UCl. His research group is active in developing nonlinear optical imaging techniques for the purpose of interrogating biological tissues and nanostructured materials.

Wendy F. Liu is an assistant professor in the Department of Biomedical Engineering and the Edwards Lifesciences Center for Advanced Cardiovascular Technology, University of California, Irvine. Her research focuses on understanding how physical and biochemical microenvironmental cues affect macrophage function, in order to develop better biomaterials for medical implants. 